CASE REPORT

\title{
Leiomyosarcomna Presenting as Abdominal Pain in a 50 Years Old Woman: A Case Report
}

\author{
*Md. Tahminur Rahman ${ }^{1}$, ss Ahmed ${ }^{2}$ \\ ${ }^{1}$ Prof. Dr. Md. Tahminur Rahman, Professor \& Head, Pathology, \\ Anwer Khan Modern Medical College, Dhanmond; \\ ${ }^{2}$ Dr. SS Ahmed, Surgeon, Dhaka Renal Center, Elephant Road, Dhaka \\ *Corresponding Author
}

\begin{abstract}
Leiomyosarcoma of uterus is a comparatively rare tumor we come across in daily histopathological examination.Clinical presentation of a leiomyosarcoma is varied and includes dysfunctional uterine bleeding,abdominal distention,abdominal pain and metastatic symptoms edepending on the organ involved like Lung, bone and brain.Here we report a case of leiomyosarcoma in a 50 year old woman having a single complain of persistent abdominal pain for last 1 year or so.Clinically and by ultrasonogram it was diagnosed as a case of large fibroid. Subsequent histopathological diagnosis was leiomyisarina. Any post menopausal woman having persistent abdominal pain and a clinical and USG diagnosis of fibroid a differential of leiomyosarcoma should be borne in mind of the physicians.
\end{abstract}

Key Words: Leiomyosarcoma, Abdominal Pain

\section{Introduction}

Leiomyosarcoma comprises of about $10-20 \%$ of soft tissue sarcomas. They occur in adults and most commonly women. The common sites are skin $^{1}$, soft tissue of extremities and retroperitoneum and uterus, cervix of women ${ }^{2,3}$. Some cases has also been reported in intramural blood vessels, renal vein and other vessels ${ }^{4,5,6}$. These leads to early metastasis. Extrauterine leiomysarcomas present as painless firm masses where as uterine leiomyosarcomas present as abdominal swelling, pain, dysfunctional uterine bleeding and metastatic masnifestations in the organs commonly involved like lungs, bone and brain. Uterine leiomyosarcoma is found equally before and after menopause and have a peak incidence at 40-60 years of age. The tumor has striking tendency to recur after removal and the 5 year survival is $40 \%$ in average. Leiomysarcomas grow within uterus in two distinctive pattern a bulky, fleshy mass projected into the uterine wall or polypoidal mass projecting in to the uterine lumen. This uncommon neoplasm arise denovo from the myometrium or endometrial stromal precursor cells. It has linked to complex variable karyotype that include frequent deletions. It is a resistant cancer, meaning generally not very responsive to chemotherapy or radiation. The best outcomes occur when it can be removed surgically with wide margins early, while small and still in situ. Other treatment options include radiation therapy, hormone therapy, and chemotherapy used either alone or in combination with another therapy $^{6}$.

Treatment recommendations depend on the specific type of sarcoma, the stage of the cancer, and the patient's sarcoma history.

A novel chemotherapy regimen that has demonstrated excellent efficacy in patients with leiomyosarcoma that has metastasized and cannot be removed surgically. The success of this regimen - a combination of gemcitabine and docetaxel - to treat leiomyosarcomas has led to additional research efforts, including two larger studies of our 
Leiomyosarcomna Presenting as Abdominal Pain in a 50 Years

treatment regimen by the Gynecologic Oncology Group, which proved the efficacy of this regimen for metastatic uterine leiomyosarcoma. This study shown that this regimen is effective in treating patients with other types of soft tissue sarcoma. For patients with early-stage leiomyosarcoma of the uterus that has been completely removed surgically, the role of adjuvant chemotherapy to reduce recurrence in women with high-risk types of the disease is also very important.

\section{Case history}

This case is a woman of 50 years of age hailing from Mirpur of Dhaka city, a house wife by profession. She is married and have a para of 3 child, the age of the last child is 33 years. She complains of persistant pain abdomen and severe weakness for last one year. She took some analgesic and antispasmodic as per doctors advice and get cured temporarily but the pain comes again. She then consulted a surgeon and on routine blood examination her hemoglobin was $6.2 \mathrm{gm} / \mathrm{dl}$ and ESR was $120 \mathrm{~mm}$ in first hour. Per vaginal examination was nothing contributoiry except some early prolapse of cervix. Ultrasonogram showed enlarged uterus with a large fibroid. A clinical diagnosis of leiomyoma was made by the physician and she was advised abdominal hysterectomy which she underwent subsequently on 22.02.2011 at a local clinic. Total abdominal hysterectomy with bilateral salpingo oophorectomy was done by spinal anaesthesia. After all aseptic precautions abdomen was opened by pfennelsteil incision. After opening a large uterus was found measuring $20 \mathrm{X} 20 \mathrm{~cm}$ in size. After successive clumping total abdominal hysterectomy with bilateral salpingo oophorectomy was done There was no adhesion. After proper haemostasis abdomen was closed in layers. Bleeding was average. She was discharged with advice with medication and rest for three months and periodic follow-up. Her uterus and both sided adnexa were sent for histopathological examination.

\section{Gross and histopathological findings}

Specimen consists of a resected uterus with cervix and both sided adnexa. The measurement of uterus with cervix is $15 \times 12 \times 10 \mathrm{~cm}$. Cut surface of uterus showed a large fibroid which occupies the whole uterine cavity measuring about $9.0 \mathrm{~cm}$ in the maximum diameter. Cut sections of the fibroid show cystic spaces containing dirty fluid and it showed focal areas of calcification, hyalinization and hemorrhage. Endometrium was not clearly discernible. The measurement of ovaries were $2.5 \mathrm{~cm}$ and $2.2 \mathrm{~cm}$ in the maximum diameter. Cut section of the both ovaries were unremarkable.

Blocks are embedded from cervix, endometrium, ovaries and fibroid.

\section{Figure: 1}

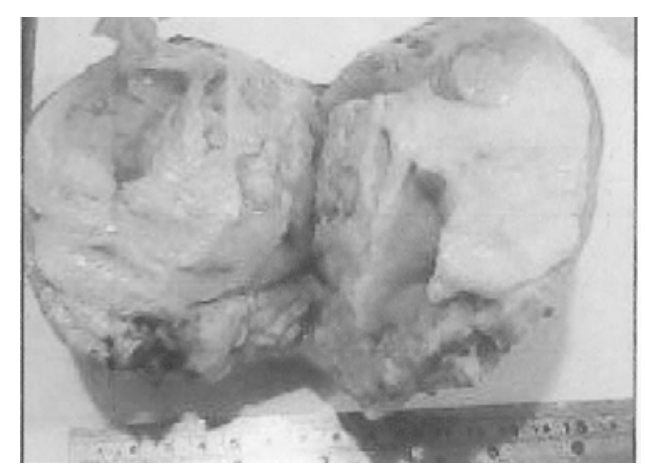

Figure I: Gross specimen of Uterus showing a leiomyosarcoma. Areas of hemorrhage, necrosis, hyalinisation are easily visible.

\section{Microscopic features}

Sections of cervix show hyperkeratosis of ectocervix consistent with prolapse. Some endocervical glands are cystically dialated containing secretions. Sections of both ovaries are unremarkable, only shows corpora albicancia. Multiple sections from grossly described fibroid show a highly cellular tumor of smooth muscle cell origin with marked nuclear atypia, increased mitotic activity (1-8/HPF). Many tumour giant cells, infarction, myxoid and hyaline degeneration and foci of dystrophic calcification are also seen. The atypical tumour cells are arranged in nodules, irregular sheets infiltrating 
the underline stroma. None of the section show any endometrial tissue. (Figure 2). A diagnosis of leiomyosarcoma was made.

Figure: 2

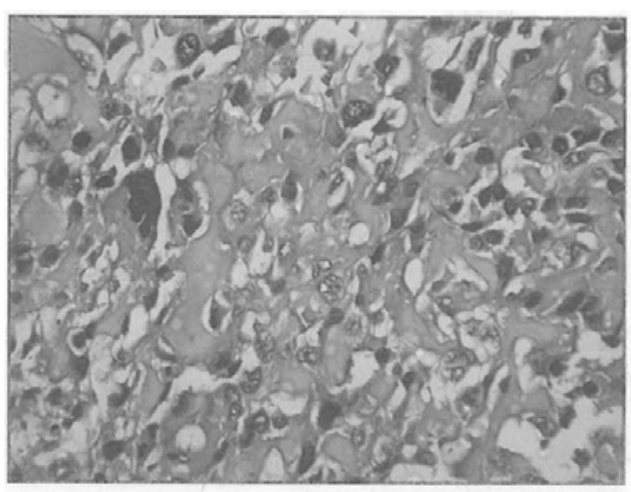

Figure II: Microphotograph of leiomyosarcoma, H\&E stain $X 40$ showing cellular atypia, pleomorphism and mitosis.

\section{Review of literatures and discussion}

Less than 4 percent of all uterine cancers are uterine sarcomas. Because uterine sarcomas are so rare and the behavior of these tumors is so variable, expert pathological review is critical. It is important for women diagnosed with these cancers to receive treatment at a center with experience caring for such patients. Uterine leiomyosarcoma is an aggressive tumour. In one retrospective series of 27 patients, there were 25 with nonmyxoid high-grade leiomyosarcoma of the uterus.

Uterine sarcomas (US) are rare and carry a poor prognosis characterized by high rates of local recurrence and metastasis. The three major histologic criteria: tumor differentiation, mitotic count, and tumor necrosis as well as other histologic and clinical factors were important and had an influence on survival and relapse . $^{8}$

Our case also showed infarction Necrosis increased mitotic count and poor tumor cell differentiation.

Forty-nine patients were diagnosed with uterine leiomyosarcoma (25 stage I, 4 stage II, 8 stage III, and 12 stage IV). Of the 49 patients, $35(71 \%)$ died of disease and 2 died of intercurrent disease. The 5 -year survival rate was $33 \%$. In a multivariate analysis with survival as the end point, stage was found to be the most important factor. All p53 immunopositive stage I-II tumors recurred within 28 months from diagnosis. This study found that stage represents the most important prognostic factor for uterine leiomyosarcomas. In stage I patients, tumor grade gives significant information regarding clinical outcome. In addition, p53 overexpression may predict a higher risk of recurrence in early stage leiomyosarcomas ${ }^{9,10}$. In other study the overall survival rate was $66 \%$ at 2 years and $57 \%$ at 5 years with a disease free survival of $54 \%$ at 2 years and $50 \%$ at 5 years. Seven patients relapsed locally and 8 developed metastases ${ }^{11}$. Another retrospective review from 1967 to 1995 of clinical and pathological characteristics of 93 patients with primary uterine sarcoma was done. The overall three-year survival rate was $67.9 \%$ and the overall five-year survival rate was $64.5 \%$. It found statistical differences ( $p<$ 0.001 ) between the stage I survival rate and other stage survival rates ${ }^{12}$.

A retrospective review of patients treated for a uterine sarcoma in Edinburgh from 1974 to 1992 has been performed. Clinical details at presentation, tumour pathology, treatment and the outcome of treatment were all recorded. 82 patients' case notes were reviewed. 54 patients had died and 28 were alive (mean follow-up period 80.3 months). 39 patients had a malignant mixed mesodermal tumour, 12 patients had an endometrial stromal sarcoma, and 27 had a leiomyosarcoma. The overall median survival is 15 months and the 5-year actuarial survival is $31 \%$. 25 of the 26 surviving patients had Stage 1 disease at presentation. Post-operative pelvic radiotherapy did not influence either survival or local tumour control. 51 of the 54 patients who relapsed had evidence of distant metastases. The conclud that total abdominal hysterectomy and bilateral salpingo-oophorectomy remains the treatment of choice for uterine sarcomas ${ }^{13}$.

Three hundred and ten cases of uterine sarcomas, among them, according to pathology: LMS (Leiomyosarcoma)-117, MMS (Mixed mesodermal sarcoma)-62, ESS (Endometrial stromal sarcoma)-56, CS (Carcinosarcoma)-27, RBMS (Rhabdomyosarcoma) 18 and other sarcomas-30, were retrospectively evaluated at the Maria Sklodowska-Curie 
Memorial Cancer Center between 1950 and 1985.Overall 5-year survival was $34.5 \%$ and in particular subgroups the survivals were as follows: the best, $52 \%$ in LMS; lower, $30 \%$ in ESS; poor survival about $20 \%$ in

\section{CS and MMS.}

A case of an epithelioid leiomyosarcoma of the uterine cervix in a 42 year-old woman was reported. This is a very rare tumor. Usually, the presenting symptoms are vaginal bleeding and abdominal pain. Two problems have to be solved by the microscopy: to prove the smooth muscle differentiation of the tumor and to assert the malignancy. Surgery remains the basis of therapy. Prognosis is poo ${ }^{14,15,16,17}$. Our case also Presented with abdominal pain only and No Vagiualbleding.

\section{Conclusions}

Any postmenopausal woman having persistent abdominal pain, discomfort apart from irregular vaginal bleeding suspected of having fibroid should undergo clinical ultrasongraphic and histopathological examination of the specimen after surgery to exclude leiomyosarcoma. Because early diagnosis bears a good prognosis and late diagnosis carries a poor prognosis to the patient in spite of surgery, chemo and radiotherapy.

\section{References}

1. Robbins pathologic Basis of Disease Editors Kumar, Abbas, Fausto, Aster. $8^{\text {th }}$ edition. 2010, New Delhi Publisher Elsevier India Pvt Limited. pp1037-38, 1254, 271.

2. Deba P Sarma, Eric E Santos and Bo Wang Leiomyosarcoma of the skin with osteoclast-like giant cells: a case report Journal of Medical Case Reports 2007, 1: 180doi: 10.1186/1752-1947-1-180

3. Van Gulik TM, Taat CW, Slors JF, Bras J, Blank LE, Bakker PJ, Kromhout JG, Brummelkamp WH. Leiomyosarcoma of large and small veins: clinical findings and results of treatment in six patients. Eur $J$ Surg Oncol 1991 Apr;17(2):125-34

4. Leu HJ, Makek M. Intramural venous leiomyosarcomas. Cancer 1986 Apr 1; 57(7):1395-400

Colombat M, Sevestre H, Gontier MF. [Epithelioid leiomyosarcoma of the uterine cervix. Report of a case]. Ann Pathol. 2001 Feb; 21(1): 48-50.

5. Hines OJ, Nelson S, Quinones-Baldrich WJ, Eilber FR. Leiomyosarcoma of the inferior vena cava: prognosis and comparison with leiomyosarcoma of other anatomic sites. Cancer 1999 Mar 1; 85(5): 1077-83

6. Soh LT, Chew SH, Ang L. Uterine leiomyosarcoma--a Singapore experience. Aust N Z J Obstet Gynaecol 1999 May; 39(2): 246-8

7. Pautier P, Genestie C, Rey A, Morice P, Roche B, Lhomme C, Haie-Meder C,Duvillard P. Analysis of clinicopathologic prognostic factors for 157 uterine sarcomas and evaluation of a grading score validated for soft tissue sarcoma. Cancer 2000 Mar 15; 88(6): 1425-31

8. Layfield LJ, Liu K, Dodge R, Barsky SH. Department of Pathology,.Uterine smooth muscle tumors: utility of classification by proliferation, ploidy, and prognostic markers versus traditional histopathology. Arch Pathol Lab Med 2000 Feb; 124(2): 221-7

9. Blom R, Guerrieri C, Stal O, Malmstrom H, Simonsen E. Leiomyosarcoma of the uterus: A clinicopathologic, DNA flow cytometric, p53, and mdm-2 analysis of 49 cases. Gynecol Oncol 1998 Jan; 68(1): 54-61

10. Coquard R, Romestaing P, Ardiet JM, Mornex F, Sentenac I, Gerard JP. Uterine sarcoma treated by surgery and postoperative radiation therapy. Patterns of relapse, prognostic factors and role of radiation therapy. Bull Cancer 1997 Jun; 84(6): 625-9

11. Gonzalez-Bosquet E, Martinez-Palones JM, GonzalezBosquet J, Garcia Jimenez A, Xercavins J. Uterine sarcoma: a clinicopathological study of 93 cases. Eur $J$ Gynaecol Oncol 1997; 18(3): 192-5.

12. Tinkler SD, Cowie VJ. Uterine sarcomas: a review of the Edinburgh experience from 1974 to 1992. : Br J Radiol 1993 Nov; 66(791): 998-1001

13. Nickie-Psikuta M, Gawrychowski K.Different types and different prognosis-study of 310 uterine sarcomas. Eur J Gynaecol Oncol 1993;14 Suppl: 105-13

14. Piura B, Rabinovich A, Yanai-Inbar I, Cohen Y, Glezerman M. Uterine sarcoma in the south of Israel: study of 36 cases J Surg Oncol 1997 Jan; 64(1): 55-62 0.

15. Olah KS, Dunn JA, Gee H. Leiomyosarcomas have a poorer prognosis than mixed mesodermal tumours when adjusting for known prognostic factors: the result of a retrospective study of 423 cases of uterine sarcoma. $\mathrm{Br} \mathrm{J}$ Obstet Gynaecol 1992 Jul; 99(7): 590-4.

16. Larson B, Silfversward C, Nilsson B, Pettersson F. Prognostic factors in uterine leiomyosarcoma. A clinical and histopathological study of 143 cases. The Radiumhemmet series 1936-1981. Acta Oncol 1990; 29(2): 185-91

17. Hannigan EV, Gomez LG. Uterine leiomyosarcoma. Am J Obstet Gynecol 1979 Jul 1; 134(5): 557-64. 